\title{
Erratum to: The fragile-to-strong dynamical crossover and the system viscoelasticity in attractive glass forming colloids
}

\author{
F. Mallamace ${ }^{1,2,3} \cdot$ C. Corsaro ${ }^{2,3} \cdot$ D. Mallamace $^{4} \cdot$ S.-H. Chen ${ }^{1}$
}

Published online: 21 September 2015

(C) Springer-Verlag Berlin Heidelberg 2015

\section{Erratum to: Colloid Polym Sci}

$$
\text { 10.1007/s00396-015-3713-6 }
$$

The original version of this article, unfortunately, contained errors. The captions to Figs. 2 and 3 were incorrect. The correct ones are as follows:

Figure 2. The temperature normalized representation of the system viscosity, $\eta(T) / \eta\left(T_{c}\right)$ vs. $T_{c} / T$. Data for $\phi=0.43,0.46,0.488$, 0.504 and 0.552 are reported. Data depicted in this figure were taken from ref. [49]. The inset shows in a scaled log-log plot the MCT power law behavior of the viscosity $\left(\eta=\eta_{0}\left|\left(T-T_{c}\right) / T_{c}\right|^{-\gamma}\right)$ for $\phi=0.256,0.43,0.46,0.488,0.504$ and 0.552 .

Figure 3. The normalized viscosity with the volume fraction as control parameter: $\eta(\phi) / \eta\left(\phi_{c}\right)$ vs. $\phi_{c} / \phi$. Data for $T=305$, $309,311,313,315$ and $318 K$ are reported. The data shown in this figure were taken from [49].

The online version of the original article can be found at http://dx.doi.org/ 10.1007/s00396-015-3713-6.

F. Mallamace

francesco.mallamace@unime.it

$\triangle$ S.-H. Chen

sowhsin@mit.edu

1 Department of Nuclear Science and Engineering, Massachusetts Institute of Technology, Cambridge, MA 02139, USA

2 CNR-IPCF Messina, Viale F. Stagno D'Alcontres 37, 98166 Messina, Italy

3 Dipartimento di Fisica e Scienze della Terra, University of Messina, Viale F. Stagno D’Alcontres 31, 98166 Messina, Italy

4 Dipartimento SASTAS, University of Messina, Viale F. Stagno D’Alcontres 31, 98166 Messina, Italy
On page 6, in the end of the section "Current state-of-the-art" before the beginning of section "Methods", the following sentence must be added:

Parts of the discussion in this paper and visualizations in figures 2 and 3 are based on our previous study of the viscosity of the system [49]. Data depicted in figures 2 and 3 were taken from ref. [49].

\section{References}

49. Mallamace F, Corsaro C, Stanley HE, Mallamace D, Chen SH (2013) The dynamical crossover in attractive colloidal systems. J Chem Phys 139:214502. doi:10.1063/1. 4833595 